\title{
ГРУППИРОВАНИЕ ЭКСПЛУАТАЦИОННЫХ ОБЪЕКТОВ НА ОСНОВЕ ПАРАМЕТРОВ ОБОБЩЁННОЙ МОДЕЛИ КРИВЫХ КАПИЛЛЯРНОГО ДАВЛЕНИЯ НА ПРИМЕРЕ РЯДА НЕФТЯНЫХ МЕСТОРОЖДЕНИЙ ЗАПАДНОЙ СИБИРИ
}

\author{
Ахметов Расуль Тухбатуллович", \\ info@of.ugntu.ru
}

\author{
Мухаметшин Вячеслав Вячеславович², \\ vv@of.ugntu.ru
}

Кулешова Любовь Сергеевна', mark1212@mail.ru

\section{Грезина Ольга Анатольевна',} ugntu.grezina@mail.ru 1 Филиал Уфимского государственного нефтяного технического университета в г. Октябрьском,
Россия, 452607, г. Октябрьский, ул. Девонская, 54а.

2 Уфимский государственный нефтяной технический университет, Россия, 450064, г. Уфра, ул. Космонавтов, 1.

\begin{abstract}
Актуальность. Группирование объектов позволяет уже на стадии выхода месторождения из разведки относить новые залежи к какому-либо из известных объектов-аналогов, находящихся в разработке. Использование опыта длительно разрабатываемых месторождений-аналогов при эксплуатации новых объектов несомненно является актуальной процедурой.

Цель: выявление объекта-аналога путем использования ограниченного количества показателей, интегрально характеризующих данный объект.

Объекты: продуктивные пласты юрского и нижнемелового возраста ряда месторождений Западной Сибири.

Методы: статистическая обработка данных капилляриметрических исследований образцов керна ряда продуктивных пластов отдельных месторождений, создание математической модели кривых капиллярного давления.

Результаты. При выявлении объектов-аналогов предлагается использовать обобщенную модель кривых капиллярного давления, которая представляет собой зависимость безразмерного капиллярного давления от нормированной (приведенной) водонасыщенности в логарифрмической системе координат. В условиях Западной Сибири для любого месторождения на коллекции образцов керна в лабораторных условиях изучают капиллярные характеристики продуктивного пласта. Oпределяют фильтрационно-емкостные свойства образцов: пористость, проницаемость, остаточную водонасьщенность. Далее путем статистической обработки лабораторных данных можно получить обобщенную математическую модель капиллярных кривых. Параметры обобщенной модели a, b и с являются характеристиками данного продуктивного пласта, причём каждый продуктивный пласт однозначно характеризуется своими параметрами. Эти параметры являются неизменными для конкретного продуктивного пласта, но различными для пластов разных месторождений. Параметр а определяет степень смачиваемости поверхности порового канала, то есть водоудерживающую способность продуктивного пласта. Параметр $b$ характеризует микронеоднородность каналов по размерам, а также долевое участие каналов в процессе фильтрации жидкости. Кроме того, относительные фазовые проницаемости для смачивающей (вода) и несмачивающей (нефрть, газ) фаз для различных водонасыщенностей тоже определяются параметром $b$. Параметр с характеризует поведение кривой капиллярного давления в области минимальных значений размеров поровых каналов. Таким образом, обобщенная модель капиллярного давления однозначно характеризует данный эксплуатационный объект, и параметры модели могут быть использованы для распознавания объекта-аналога, находящегося в разработке. Очевидно, опыт разработки объекта-аналога можно уверенно использовать в процессе разработки рассматриваемого месторождения.
\end{abstract}

\section{Ключевые слова:}

Кривая капиллярного давления, обобщенная модель, группирование объектов, капилляриметрические исследования, фильтрационно-емкостные свойства.

\section{Введение}

Группирование используется для решения важнейших задач нефтепромысловой геологии и разработки нефтяных и газовых месторождений. Это выяснение сходства и различия продуктивных пластов при выделении эксплуатационных объектов; обоснование системы разработки, выбор мероприятий по контролю и регулированию разработки и повышению нефтеизвлечения [1-6].

Группирование заключается в выделении относительно однородных групп по комплексу параметров (признаков), характеризующих генеральную совокупность объектов.

Комплекс параметров, используемых при группировании объектов, включает фильтрационноемкостные свойства, микро- и макронеоднородность, условия залегания продуктивных пластов, а также физико-химические свойства пластовых флюидов и т. д. [7-10].

При этом наибольший интерес представляют алгоритмы группирования по ограниченному количеству параметров, поскольку они позволяют уже на 
стадии выхода месторождения из разведки и составления первых проектных документов по разработке относить новые объекты к какой-либо из известных групп объектов-аналогов, находящихся в разработке [11-13].

Использование опыта длительно разрабатываемых месторождений-аналогов позволяет в условиях новых объектов принять обоснованные технологические решения при составлении проектов разработки, наметить пути и способы повышения эффективности разработки, правильно и обоснованно осуществлять контроль и регулирование процесса разработки [14-17].

\section{Материалы и методы}

Очевидно, группирование месторождений по ограниченному количеству признаков возможно лишь при использовании обобщенных параметров, интегрально характеризующих данный объект. Другими словами, обобщенные параметры должны быть практически неизменными для данного конкретного месторождения и однозначно характеризовать данный эксплуатационный объект.

По нашему мнению, обобщенные параметры могут быть выявлены путем анализа результатов лабораторного моделирования фильтрации флюидов, например, в процессе капилляриметрических исследований образцов керна из продуктивного пласта.

Действительно при капилляриметрии моделируются процессы движения флюидов, соответствующие процессу образования месторождений нефти и газа.

Капиллярная кривая графически изображается, как правило, в виде функции капиллярного давления от водонасыщенности [18].

В работах $[19,20]$ предлагается в качестве коррелирующей функции в условиях коллекторов Западной Сибири использовать алгебраический многочлен второй степени в логарифмической системе координат, который позволяет с высокой точностью аппроксимировать экспериментальный график зависимости капиллярного давления от нормированной водонасыщенности.

На рис. 1, 2 представлены графики зависимости логарифма безразмерного капиллярного давления $\left(P_{\mathrm{\kappa}} \cdot r_{0}\right)$ от логарифма нормированной водонасыщенности $K_{\mathrm{B}}^{*}$ для нескольких месторождений Западной Сибири.

Из анализа представленных графиков (рис. 1, 2) можно сделать следующие выводы:

1. Линия регрессии, полученная на основе результатов капилляриметрических исследований на коллекции образцов керна, полностью характеризует данный продуктивный пласт, а именно его фильтрационно-емкостные, а также физикохимические свойства поверхности пустотного пространства породы.

2. Зависимости, представленные на рис. 1, 2, являются графическим «образом» данного продуктивного пласта, т. е. форма и расположение графиков на координатной плоскости практически полностью характеризуют данный пласт-коллектор.
Исходя из вышеизложенного, поиск объектааналога заключается в сопоставлении графических «образов» данного пласта с другими объектами. При этом идеальный случай соответствует полному совпадению графических образов.

Задача группирования заключается в распознавании объекта, находящегося в разработке, графический образ которого имеет минимальное отклонение от графического образа данного объекта.

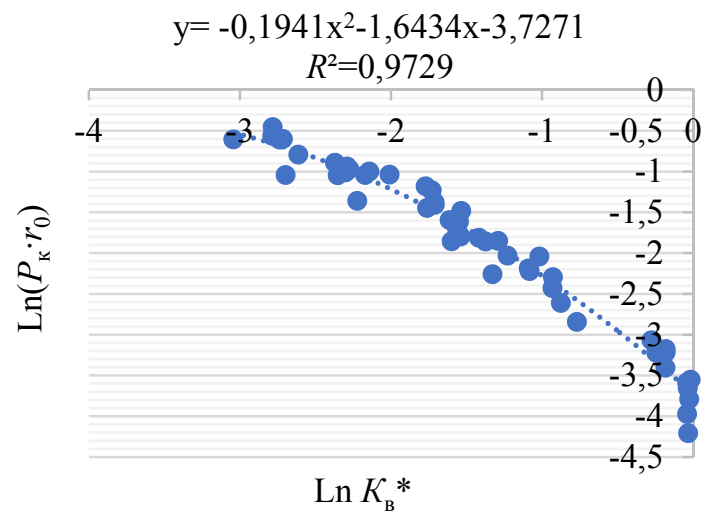

Pис. 1. Зависимость нормированной водонасыщенности от безразмерного капиллярного давления в логарифмической системе координат. Лас-Еганское месторождение, пласт $A B_{1-3}$

Fig. 1. Dependence of the normalized water saturation on the dimensionless capillary pressure in the logarithmic coordinate system. Las-Eganskoe field, $A V_{l-3}$ formation

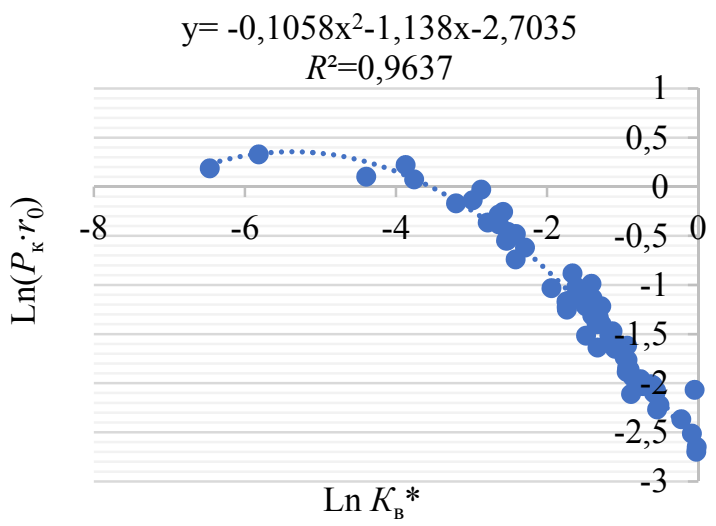

Pис. 2. Зависимость нормированной водонасыщенности от безразмерного капиллярного давления в логарифмической системе координат. Повховское месторождение, пласт ЮВ

Fig. 2. Dependence of the normalized water saturation on the dimensionless capillary pressure in the logarithmic coordinate system. Povkhovskoe field, $J V_{1-1}$ formation

\section{Результаты и обобщение}

Рассмотрим аналитическую модель капиллярных кривых, т. е. математическое описание графического объекта продуктивного пласта. Она содержит комплекс обобщенных параметров, необходимых для группирования объектов разработки. 
В работе [19] представлена обобщённая модель капиллярных кривых для коллекторов Западной Сибири:

$$
\ln \left(P r_{0}\right)=a+b \cdot \ln \left(K_{\mathrm{B}}^{*}\right)+c \cdot\left(\ln \left(K_{\mathrm{B}}^{*}\right)\right)^{2},
$$

где $a, b, c-$ фиксированные параметры; $K_{\mathrm{в}}^{*}=\frac{K_{\mathrm{B}}-K_{\mathrm{вo}}}{1-K_{\text {во }}}-$ нормированная водонасыщенность порового пространства; $K_{\text {в }}$ - общая водонасыщенность; $K_{\text {во }}$ - остаточная водонасыщенность; $r_{0}=\sqrt{\frac{K_{\text {пр }}}{K_{\text {п }}}}-$ параметр, имеющий размерность радиуса; $K_{\text {пр }}$ - коэффициент абсолютной проницаемости, мкм ${ }^{2} ; K_{\text {п }}-$ коэффициент пористости; $P$ - капиллярное давление, атм.

Рассмотрим физический смысл параметров, входящих в обобщенную модель кривых капиллярного давления.

Определим из формулы (1) начальные капиллярные давления $\left(P_{0}\right)$. Для этого в формулу $(1)$ подставим $K_{\mathrm{B}}^{*}=1,0$.

Мы получим: $\ln \left(P_{0} r_{0}\right)=a$. Отсюда:

$$
P_{0}=\frac{\mathrm{e}^{\mathrm{a}}}{r_{0}}=\frac{\mathrm{e}^{\mathrm{a}}}{\sqrt{\frac{K_{\mathrm{mp}}}{K_{\mathrm{n}}}}} .
$$

Очевидно, начальное капиллярное давление $P_{0}$ (формула (2)) соответствует максимальному радиусу поровых каналов образца породы $r_{\mathrm{m}}$.

Действительно, в соответствии с формулой Лапласа, имеем:

$$
r_{\mathrm{m}}=\frac{2 \sigma \cos \theta}{P_{0}}=2 \sigma \cos \theta \cdot \mathrm{e}^{-\mathrm{a}} \sqrt{\frac{K_{\text {пр }}}{K_{\text {п }}}},
$$

где $\sigma$ - величина поверхностного натяжения; $\theta$ - угол смачивания.

В условиях Западной Сибири для любого месторождения на коллекции образцов керна в лабораторных условиях изучают капиллярные характеристики продуктивного пласта. Кроме того, определяют фильтрационно-емкостные свойства образцов: пористость, проницаемость, остаточную водонасыщенность [20-22].

Далее путем статистической обработки лабораторных данных можно получить выражение для математической модели капиллярных кривых данного месторождения в виде формулы (1).

Заметим, что параметры $a, b$ и $c$ являются характеристиками данного продуктивного пласта. Причем каждый продуктивный пласт характеризуется своими параметрами.

Обратимся к формуле (3). В соответствии с этой формулой для разных месторождений при одинаковых значениях пористости и проницаемости максимальные радиусы поровых каналов различаются. Отметим, что различаются не только максимальные, но и средние значения радиусов поровых каналов.

Более глубокий анализ показывает, что множитель $\mathrm{e}^{-\mathrm{a}}$ в формуле (3) определяет степень смачиваемости поверхности поровых каналов, т. е. водоудерживающую способность
В работе [19] нами получена связь между параметрами $\sqrt{\frac{K_{\text {пр }}}{K_{\text {п }}}}$ и $\frac{1-K_{\text {во }}}{K_{\text {во }}}$ в следующем виде:

$$
\sqrt{\frac{K_{\text {пр }}}{K_{\text {п }}}}=\left(\delta \frac{\mathrm{e}^{\mathrm{a}}}{\sigma \cos \theta} \cdot \frac{\alpha+2}{\alpha-1}\right) \frac{1-K_{\text {во }}}{K_{\text {во }}},
$$

где $\delta$ - толщина пленки остаточной воды.

Проанализируем данное выражение.

Левая часть формулы (4) содержит абсолютную проницаемость и пористость образца. Оба этих параметра определяются в лабораторных условиях на сухих (безводных) образцах керна.

Правая часть формулы (4) содержит остаточную водонасыщенность, которая определяется в образцах, насыщенных водой.

Таким образом, выражение, находящееся в скобках, осуществляет связь между свойствами образца в сухом (безводном) и водонасыщенном состояниях. Ясно, что это выражение, содержащее множитель $\mathrm{e}^{-\mathrm{a}}$, учитывает фактор смачиваемости водой поверхности поровых каналов.

Таким образом, параметр $\mathrm{e}^{-\mathrm{a}}$, являющийся неизменным для конкретного продуктивного пласта, но различным для разных месторождений, характеризует фактор смачиваемости водой данного продуктивного пласта.

Теперь выясним физический смысл параметра $b$ в формуле (1).

В работе [23] показано, что если пренебречь третьим членом в формуле (1), то получим следующую простую формулу для приведённой водонасыщенности:

$$
K_{\mathrm{B}}^{*}=\left(\frac{P}{P_{0}}\right)^{\frac{1}{b}} .
$$

Данная формула (5) представляет собой приближение Брукса-Кори для аппроксимации капиллярных кривых

Далее воспользуемся формулой Лапласа и перейдём к радиусам поровых каналов

$$
K_{\mathrm{B}}^{*}=\left(\frac{r}{r_{\mathrm{m}}}\right)^{\alpha},
$$

где $\alpha=\frac{1}{b}$.

Теперь вычислим производную по радиусу и получим плотность распределения поровых каналов по размерам:

$$
g(r)=\frac{\mathrm{d} K_{\mathrm{B}}^{*}}{\mathrm{~d} r}=\frac{\alpha}{r_{\mathrm{m}}^{\alpha}} r^{\alpha-1},
$$

где $\alpha$ - крутизна капиллярных кривых; $r_{\mathrm{m}}$ - максимальный радиус поровых каналов.

Ниже представлены выражения для дисперсии (Д) и коэффициента вариации $(W)$ размеров поровых каналов:

$$
Д=\frac{\alpha}{\alpha+2} r_{\mathrm{m}}^{2}, \quad W=\frac{1}{\alpha(\alpha+2)} .
$$


Таким образом, дисперсия и коэффициент вариации размеров поровых каналов определяется параметром $\alpha$ или крутизной капиллярных кривых. Отсюда следует, что параметр характеризует микронеоднородность поровых каналов по размерам.

Наши исследования показывают, что параметр $b$ определяет не только распределение поровых каналов по размерам, но и их долевое участие в фильтрации жидкости.

Кроме того, относительные фазовые проницаемости для смачивающей (вода) и несмачивающей (нефть, газ) фаз для различных водонасыщенностей порового пространства также определяются параметром $b$.

Рассмотрим физический смысл параметра $c$ обобщенной модели.

Очевидно, параметр $c$ характеризует поведение кривой капиллярного давления при минимальных значениях приведённой водонасыщенности, т. е. вблизи вертикальной асимптоты, соответствующей резкому возрастанию капиллярного давления. Это область минимальных значений размеров поровых каналов.

Как было указано выше, значения коэффициентов $a, b$ и $c$ определяются путем статистической обработки данных лабораторных капилляриметрических исследований образцов керна из продуктивного горизонта на стадии подсчета запасов.

В табл. 1 представлены значения коэффициентов $a$, $b$ и $c$ для различных пластов ряда месторождений Западной Сибири.

Анализ данных табл. 1 показывает, что параметры обобщенной модели капиллярных кривых для рассматриваемых месторождений изменяются в следующих пределах (табл. 2).

На рис. 3 представлены линии регрессии для зависимостей безразмерного капиллярного давления от нормированной водонасыщенности в логарифмической системе координат для отдельных продуктивных пластов ряда месторождений. Видно, что линии регрессии для разных объектов существенно различаются.

Совокупность объектов, находящихся в разработке, для которых путём обработки данных капиллярных исследований получены уравнения обобщенной модели, назовём базовой совокупностью.

Допустим, что вводится в эксплуатацию новый объект. Для этого объекта уже на стадии составления первых проектных документов мы имеем результаты лабораторных капиллярных исследований, а также данные определения фильтрационно-емкостных свойств продуктивного пласта.

При этом на основе данных лабораторных исследований образцов керна из продуктивного пласта нового месторождения можно составить обобщенную модель кривых капиллярного давления. Далее путем визуального сопоставления графика с линиями регрессии для пластов соседних месторождений (базовая совокупность объектов) можно найти объектаналог, находящийся в разработке.
Таблица 1. Значения параметров обобщенной модели

Table 1. Generalized model parameter values

\begin{tabular}{|c|c|c|c|c|}
\hline \multirow{2}{*}{$\begin{array}{c}\text { Месторождение } \\
\text { Field } \\
\end{array}$} & \multirow{2}{*}{$\begin{array}{l}\text { Пласт } \\
\text { Layer } \\
\end{array}$} & \multicolumn{3}{|c|}{ Параметр/Parameter } \\
\hline & & $a$ & $b$ & $c$ \\
\hline $\begin{array}{l}\text { Урьевское } \\
\text { Urievskoe } \\
\end{array}$ & $\begin{array}{l}\mathrm{AB}_{1-3} \\
\mathrm{AV}_{1-3}\end{array}$ & $-3,05$ & $-1,744$ & $-0,164$ \\
\hline $\begin{array}{l}\text { Повховское } \\
\text { Povkhovskoe }\end{array}$ & $\begin{array}{c}\mathrm{FB}_{1-1} \\
\mathrm{JV}_{1-1}\end{array}$ & $-2,76$ & $-1,1825$ & $-0,1122$ \\
\hline $\begin{array}{c}\text { Андреевское } \\
\text { Andreevskoe }\end{array}$ & $\begin{array}{l}\Pi \\
\mathrm{P} \\
\end{array}$ & $-3,0082$ & $-1,7956$ & $-0,4034$ \\
\hline \multirow{3}{*}{$\begin{array}{l}\text { Северо-Поточное } \\
\text { Severo-Potochnoe }\end{array}$} & $\begin{array}{l}\mathrm{LB}_{6} \\
\mathrm{BV}_{6}\end{array}$ & $-4,5812$ & $-2,6799$ & $-0,2873$ \\
\hline & $\begin{array}{l}\mathrm{BB}_{6} \\
\mathrm{BV}_{6}\end{array}$ & $-2,0565$ & $-0,6824$ & 0,1125 \\
\hline & $\begin{array}{l}\mathrm{BB}_{10} \\
\mathrm{BV}_{10}\end{array}$ & $-1,7492$ & $-0,419$ & 1,222 \\
\hline $\begin{array}{l}\text { Новоортъягунское } \\
\text { Novoortyagunskoe }\end{array}$ & $\begin{array}{c}\mathrm{OB}_{1} \\
\mathrm{JV}_{1}\end{array}$ & $-2,606$ & $-1,753$ & 0,5608 \\
\hline $\begin{array}{l}\text { Пайтыхское } \\
\text { Paytykhskoe } \\
\end{array}$ & $\begin{array}{l}\mathrm{OB}_{4} \\
\mathrm{JV}_{4}\end{array}$ & $-3,9823$ & $-4,3451$ & $-0,2883$ \\
\hline $\begin{array}{l}\text { Равенское } \\
\text { Ravenskoe }\end{array}$ & $\begin{array}{c}\mathrm{OC}_{2} \\
\mathrm{JS}_{2}\end{array}$ & $-0,3151$ & $-0,805$ & $-0,0153$ \\
\hline \multirow{4}{*}{$\begin{array}{l}\text { Лас-Еганское } \\
\text { Las-Eganskoe }\end{array}$} & $\begin{array}{l}\mathrm{AB}_{1-3} \\
\mathrm{AV}_{1-3}\end{array}$ & $-3,6083$ & $-1,5$ & $-0,1564$ \\
\hline & $\begin{array}{l}\mathrm{AB}_{2} \\
\mathrm{AV}_{2}\end{array}$ & $-2,4662$ & $-1,2836$ & $-0,1684$ \\
\hline & $\begin{array}{l}\mathrm{BB}_{6} \\
\mathrm{BV}_{6}\end{array}$ & $-2,9074$ & $-1,4115$ & $-0,1411$ \\
\hline & $\begin{array}{l}\mathrm{bB}_{8} \\
\mathrm{BV}_{8} \\
\end{array}$ & $-2,924$ & $-1,4212$ & $-0,1249$ \\
\hline \multirow{3}{*}{$\begin{array}{l}\text { Каменное } \\
\text { Kamennoe }\end{array}$} & $\begin{array}{l}\mathrm{BK}_{1} \\
\mathrm{VK}_{1}\end{array}$ & $-3,9321$ & $-1,9009$ & $-0,2471$ \\
\hline & $\begin{array}{l}\mathrm{BK}_{2} \\
\mathrm{VK}_{2} \\
\end{array}$ & $-3,6273$ & $-1,7365$ & $-0,2046$ \\
\hline & $\begin{array}{l}6 B_{3} \\
\mathrm{BV}_{3} \\
\end{array}$ & $-3,4416$ & $-1,6499$ & $-0,1679$ \\
\hline \multirow{2}{*}{$\begin{array}{l}\text { Ловинское } \\
\text { Lovinskoe }\end{array}$} & $\begin{array}{c}\mathrm{O}_{2-4} \\
\mathrm{~J}_{2-4} \\
\end{array}$ & $-3,0258$ & $-1,2514$ & $-0,1644$ \\
\hline & $\begin{array}{l}\mathrm{OB}_{5-6} \\
\mathrm{JV}_{5-6}\end{array}$ & $-3,4468$ & $-1,4133$ & $-0,1419$ \\
\hline
\end{tabular}

Таблица 2. Предель изменения значений параметров обобшенной модели

Table 2. Limits of parameter values of the generalized model variation

\begin{tabular}{|c|c|c|}
\hline \multirow{2}{*}{$\begin{array}{l}\text { Параметр } \\
\text { Parameter }\end{array}$} & \multicolumn{2}{|c|}{ Пределы изменения/Variation limits } \\
\cline { 2 - 3 } & Минимальное/min & Максимальное/max \\
\hline$a$ & $-4,58$ & $-0,135$ \\
\hline$b$ & $-5,58$ & $-0,42$ \\
\hline$c$ & $-0,40$ & $+1,22$ \\
\hline
\end{tabular}

Очевидно, опыт разработки объекта-аналога можно уверенно использовать в процессе разработки нового месторождения [24-28].

Наибольший интерес представляют аналитические методы распознавания аналога.

Легко заметить, что уравнения регрессии могут быть представлены в виде векторов с началом, совпадающим с началом трехмерной декартовой системы координат

$$
\bar{y}=\{a, b, c\},
$$

где $a, b, c$ - компоненты вектора.

Пусть новый объект характеризуется вектором $\overline{y_{0}}=\left\{a_{0}, b_{0}, c_{0}\right\}$, а разрабатываемые объекты соответственно векторами $\overline{y_{\mathrm{i}}}=\left\{a_{\mathrm{i}}, b_{\mathrm{i}}, c_{\mathrm{i}}\right\}$, где $i=1-N, N$ - общее число разрабатываемых объектов. 


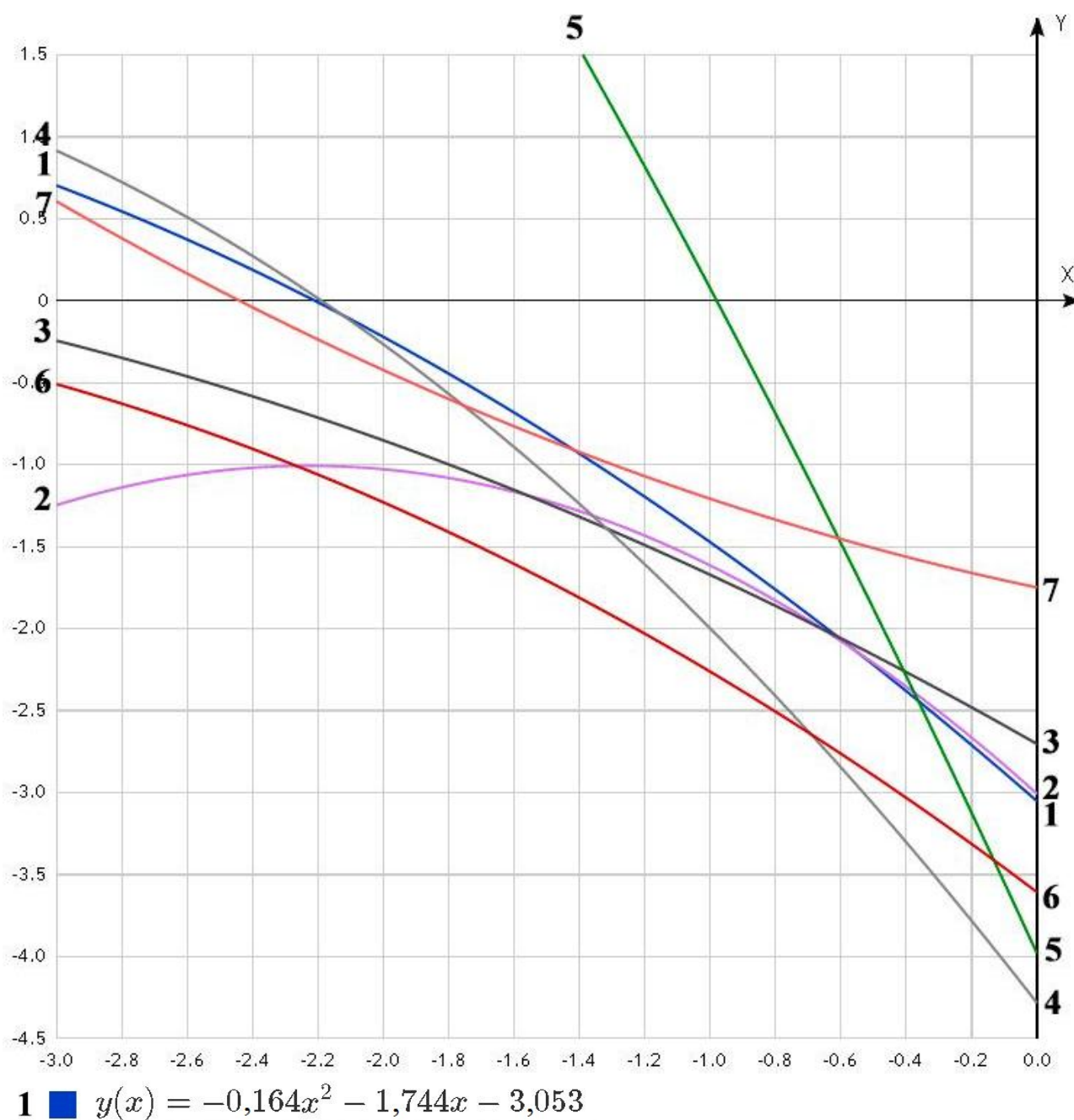

1 마 $y(x)=-0,164 x^{2}-1,744 x-3,053$

$2 \quad y(x)=-0,403 x^{2}-1,796 x-3,008$

$3 \square y(x)=-0,106 x^{2}-1,138 x-2,704$

$4 \quad y(x)=-0,276 x^{2}-2,561 x-4,284$

$5 \square y(x)=-0,288 x^{2}-4,345 x-3,982$

$6 \square y(x)=-0,156 x^{2}-1,501 x-3,608$

$7 \square y(x)=0,122 x^{2}-0,419 x-1,749$

Рис. 3. Линии регрессии зависимостей безразмерного капиллярного давления от нормированной водонасыщенности для ряда объектов Западной Сибири

Fig. 3. Regression lines for dependences of dimensionless capillary pressure on normalized water saturation for a number of facilities in Western Siberia

Очевидно, для выявления объекта-аналога необходимо сопоставить числовые значения компонентов векторов нового объекта и базовой совокупности объектов.

Идеальный случай - равенство соответствующих компонентов векторов.

В общем случае задача сводится к минимизации суммы квадратов разностей соответствующих компонент:

$$
R^{2}=\left(a_{\mathrm{i}}-a_{0}\right)^{2}+\left(b_{\mathrm{i}}-b_{0}\right)^{2}+\left(c_{\mathrm{i}}-c_{0}\right)^{2}=\min ,
$$

где $R^{2}$ - квадрат расстояния между концами нового вектора и искомого вектора-аналога.
В заключение рассмотрим порядок выявления объекта-аналога с использованием обобщенной модели:

- в лабораторных условиях на образцах керна данного объекта проводятся капилляриметрические исследования, а также определяются открытая пористость, абсолютная проницаемость и остаточная водонасыщенность;

- путем статистической обработки данных получают обобщенную модель кривых капиллярного давления;

- в соответствии с формулой (6) параметры обобщенной модели сопоставляются с соответствую- 
щими параметрами базовой совокупности объектов и находят объект-аналог, для которого выполняется условие минимизации квадрата расстояния $\left(R^{2}\right)$.

\section{Выводы}

1. Обобщенная математическая модель капиллярных кривых однозначно характеризует эксплуатационный объект месторождения.

\section{СПИСОК ЛИТЕРАТУРЫ}

1. Сергеев В.Л., Нгуен Ф.Т.Х. Модели и алгоритмы адаптивной интерпретации результатов комбинированных газогидродинамических исследований интеллектуальных скважин // Известия Томского политехнического университета. Инжиниринг георесурсов. - 2018. - Т. 329. - № 10. - С. 67-75.

2. Мухаметшин В.В. Повышение эффективности управления объектами добычи нефти с использованием метода аналогий // SOCAR Proceedings. - 2020. - № 4. - C. 42-50. DOI: 10.5510/OGP20200400464.

3. Feng Zhang, Daoyong Yang. Determination of minimum permeability plateau and characteristic length in porous media with non-Darcy flow behavior // Journal of Petroleum Science and Engineering. - 2014. - V. 119. - P. 8-16. DOI: 10.1016/j.petrol.2014.04.018

4. Artificial neural network-based time-domain interwell tracer testing for ultralow-permeability fractured reservoirs / Cheng Jing, Xiaowei Dong, Wenhao Cuid, Zhenzhen Dong, Long Rena, Xiaoyu Gua, Yanlong He, Chunsheng Pu, Xiaoyu Liu // Journal of Petroleum Science and Engineering. - 2020. - V. 195. - 107558. P. 1-9. DOI: 10.1016/j.petrol.2020.107558

5. Khormali A., Sharifov A.R., Torba D.I. Investigation of barium sulfate precipitation and prevention using different scale inhibitors under reservoir conditions // International Journal of Engineering, Transactions A: Basics. - 2018. - V. 31. - Iss. 10. - P. 1796-1802. DOI: $10.5829 /$ ije.2018.31.10a.24.

6. Рогачев М.К., Мухаметшин В.В., Кулешова Л.С. Повышение эффективности использования ресурсной базы жидких углеводородов в юрских отложениях Западной Сибири // Записки Горного института. - 2019. - Т. 240. - С. 711-715. DOI 10.31897/PMI.2019.6.711.

7. Рогачев М.К., Мухаметшин В.В. Контроль и регулирование процесса солянокислотного воздействия на призабойную зону скважин по геолого-промысловым данным // Записки Горного института. - 2018. - T. 231. - C. 275-280. DOI: 10.25515/PMI.2018.3.275.

8. Use of hydrochloric acid to remove filter-cake damage from preformed particle gel during conformance-control treatments A. Imqam, B. Bai, M. Wei, H. Elue, F.A. Muhammed // SPE Production \& Operations. - 2016. - V. 31. - № 3. - 11 p. DOI 10.2118/172352-PA.

9. Новый подход к оценке результатов гидравлического разрыва пласта (на примере бобриковской залежи Шершневского месторождения) / В.И. Галкин, И.Н. Пономарева, С.С. Черепанов, Е.В. Филиппов, Д.А. Мартюшев // Известия Томского политехнического университета. Инжиниринг георесурсов. 2020. - T. 331. - № 4. - C. 107-114. DOI: $10.18799 / 24131830 / 2020 / 4 / 2598$.

10. Evolution of anisotropic permeability of fractured sandstones subjected to true-triaxial stresses during reservoir depletion Tongwen Jiang, Wei Yao, Xiongwei Sun, Chunyan Qi, Xing Li, Kaiwen Xia, Jie Zhang, M.H.B. Nasseri // Journal of Petroleum Science and Engineering. - 2020. - 108251. - P. 1-17. DOI 10.1016/j.petrol.2020.108251

11. Козырев Н.Д., Вишняков А.Ю., Путилов И.С. Оценка влияния параметров неопределенности на прогнозирование показателей разработки // Недропользование. - 2020. - Т. 20. - № 4. C. 356-368. DOI: $10.15593 / 2712-8008 / 2020 / 4 / 5$.

12. Мухаметшин В.В., Кулешова Л.С. О снижении уровня неопределенности при управлении заводнением залежей с трудноизвлекаемыми запасами // Известия Томского политехниче-
2. Параметры обобщенной модели могут быть использованы при группировании эксплуатационных объектов.

3. Поиск объекта-аналога сводится к сопоставлению одноименных параметров нового объекта с параметрами соседних объектов, находящихся в разработке. При этом в качестве аналога выбирается объект с такими же или близкими значениями параметров.

ского университета. Инжиниринг георесурсов. - 2020. T. 331. - № 5. - C. 140-146. DOI 10.18799/24131830/2020/5/2644

13. Formation permeability evaluation and productivity prediction based on mobility from pressure measurement while drilling / Sh.I. Xinlei, C.U.I. Yunjiang, X.U. Wankun, Zhang Jiansheng, Guan Yeqin // Petroleum Exploration and Development. - 2020. V. 47. - Iss. 1. - P. 146-153. DOI: 10.1016/S18763804(20)60013-1.

14. Optimization of surfactant-based fluids for acid diversion H.A. Nasr-El-Din, M.S. Van Domelen, L. Sierra, Th.D. Welton // European Formation Damage Conference. - Scheveningen, The Netherlands, 30 May - 1 June 2007. - 11 p. DOI: 10.2118/107687-MS

15. Оптимизация выработки запасов из водонефтяных зон горизонта D3ps Шкаповского нефтяного месторождения с помощью горизонтальных скважин / Р.Ф. Якупов, В.Ш. Мухаметшин, И.Н. Хакимзянов, В.Е. Трофимов // Георесурсы. 2019. - T. 21. - № 3. - C. 55-61. DOI: 10.18599/grs.2019.3.55-61.

16. Ghareb Hamada, Veronique Joseph. Developed correlations between sound wave velocity and porosity, permeability and mechanical properties of sandstone core samples // Petroleum Research. - 2020. - V. 5. - Iss. 4. - P. 326-338. DOI: 10.1016/j.ptlrs.2020.07.001.

17. Akhmetov R.T., Kuleshova L.S., Mukhametshin V.V. Application of the Brooks-Corey model in the conditions of lower cretaceous deposits in terrigenous reservoirs of Western Siberia // IOP Conference Series: Materials Science and Engineering (MEACS 2018 - International Conference on Mechanical Engineering, Automation and Control Systems). - 2019. - V. 560 - 012004 P. 1-4. DOI: 10.1088/1757-899X/560/1/012004

18. Application of X-ray computed tomography for the virtual permeability prediction of fiber reinforcements for liquid composite molding processes: a review / M.A. Ali, R. Umer, K.A. Khan, W.J. Cantwell // Composites Science and Technology. - 2019. - V. 184. - 107828. - P. 1-20. DOI: 10.1016/j.compscitech.2019.107828.

19. Jun He, Kegang Ling. Measuring permeabilities of Middle-Bakken samples using three different methods // Journal of Natural Gas Science and Engineering. - 2016. - V. 31. - P. 28-38. DOI: 10.1016/j.jngse.2016.03.007

20. The generalized correlating function of capillary curves and the relationship of the filtration-capacitive parameters of reservoirs in Western Siberia with the size distribution of pore channels / R.T. Akhmetov, V.V. Mukhametshin, L.S. Kuleshova, O.A. Grezina, P.M. Malyshev // Journal of Physics: Conference Series (International Conference on Information Technology in Business and Industry (ITBI 2020)). - 2020. - V. 1661. 012016. - P. 1-7. DOI: 10.1088/1742-6596/1661/1/012016.

21. Михайлов Н.Н., Сечина Л.С., Моторова К.А. Роль глинистых минералов в образовании адсорбционно-связанной нефти в породах-коллекторах углеводородного сырья // Георесурсы, геоэнергетика, геополитика. - 2012. - № 1 (5). - С. 51-62.

22. Шелепов В.В., Тюкавкина О.В. Геофизические методы определения коллекторских свойств сложнопостроенных нижнесреднеюрских залежей в пределах месторождений широтного Приобья // Вестник Московского университета. Серия 4: Геология. - 2020. - № 1. - C. 37-41. DOI: $10.3103 / \mathrm{S} 0145875220020088$.

23. Диагностические критерии выделения фильтрационных потоков в процессе гидродинамических исследований горизонтальных скважин / В.Л. Сергеев, В.Х. Донг, Д.Э. Хагай, А.В. Игнатенко // Известия Томского политехнического уни- 
верситета. Инжиниринг георесурсов. - 2020. - Т. 331. - № 2. C. $181-187$. DOI: $10.18799 / 24131830 / 2020 / 2 / 2504$

24. Alimkhanov R., Samoylova I. Application of data mining tools for analysis and prediction of hydraulic fracturing efficiency for the BV8 reservoir of the Povkh oil field // SPE Russian oil and gas exploration \& Production technical conference and exhibition. Moscow, Russia, 14-16 October 2014. - 20 p. DOI: 10.2118/171332-RU.

25. Soloviev N.N., Mukhametshin V.Sh., Safiullina A.R. Developing the efficiency of low-productivity oil deposits via internal flooding // IOP Conference Series: Materials Science and Engineering (International Conference on Extraction, Transport, Storage and Processing of Hydrocarbons \& Materials (ETSaP)). - 2020. V. 952. - 012064. - P. 1-5. DOI: 10.1088/1757-899X/952/1/012064.
26. Interference well-test model for vertical well with double-segment fracture in a multi-well system / Jiazheng Qin, Shiqing Cheng, Peng Li, Youwei He, Xin Lu, Haiyang Yu // Journal of Petroleum Science and Engineering. - 2019. - V. 183. - 106412. - P. 1-14. DOI: $10.1016 /$ j.petrol.2019.106412.

27. Сметкина М.А., Мелкишев О.А., Присяжнюк М.А. Уточнение значений проницаемости при адаптации гидродинамической модели // Недропользование. - 2020. - Т. 20. - № 3. - С. 223-230. DOI: $10.15593 / 2712-8008 / 2020.3 .3$.

28. McLachlan G.J. Discriminant analysis and statistical pattern recognition. - Hoboken, New Jersey: John Wiley \& Sons, 2004. $545 \mathrm{p}$.

Поступила 21.10.2021 2.

\section{Информация об авторах}

Axметов P.T., кандидат технических наук, профессор кафедры разведки и разработки нефтяных и газовых месторождений, филиал Уфимского государственного нефтяного технического университета в г. Октябрьском.

Мухаметшин B.B., доктор технических наук, профессор кафедры разведки и разработки нефтяных и газовых месторождений, Уфимский государственный нефтяной технический университет.

Кулешова Л.С., кандидат технических наук, старший преподаватель кафедры разведки и разработки нефтяных и газовых месторождений, заместитель директора по УР, филиал Уфимского государственного нефтяного технического университета в г. Октябрьском.

Грезина О.A., доцент, старший преподаватель, заместитель заведующего кафедрой разведки и разработки нефтяных и газовых месторождений, филиал Уфимского государственного нефтяного технического университета в г. Октябрьском. 
UDC 622.276 .63

\title{
PRODUCTION FACILITIES GROUPING BASED ON THE PARAMETERS OF THE CAPILLARY PRESSURE CURVES GENERALIZED MODEL ON THE EXAMPLE OF WESTERN SIBERIA OIL FIELDS
}

\author{
Rasul T. Akhmetov', \\ info@of.ugntu.ru
}

\author{
Vyacheslav V. Mukhametshin², \\ vv@of.ugntu.ru
}

Lyubov S. Kuleshova1,
markl212@mail.ru

Olga A. Grezina', ugntu.grezina@mail.ru
1 Ufa State Petroleum Technological University, Branch of the University in the City of Oktyabrsky, 54a, Devonskaya street, Oktyabrsky, 452607, Russia.
2 Ufa State Petroleum Technological University,
1, Kosmonavtov street, Ufa, 450064, Russia.

\begin{abstract}
The relevance. The grouping of facilities allows, already at the stage of the field exit from exploration, classifying new deposits as any of the known analogue one being in development. Using the experience of long-term developed analogous fields in the exploitation of new facilities is undoubtedly a relevant procedure.

The purpose: identification of an analogous facility by employing a limited number of indicators integrally characterizing the facility in question.

The objects: productive strata of the Jurassic and Lower Cretaceous age of a number of fields in Western Siberia.

Methods: statistical data processing of core samples capillarimetric studies from a number of productive formations of individual fields, a mathematical model of capillary pressure curves creation.

Results. When identifying analogous objects, it is proposed to use a generalized model of capillary pressure curves, which is the dependence of the dimensionless capillary pressure on the normalized (reduced) water saturation in a logarithmic coordinate system. For any field in the conditions of Western Siberia the capillary characteristics of the productive formation are studied on the laboratory core samples collection. The filtration-capacity properties of the samples: porosity, permeability, residual water saturation, are determined. Further, by the laboratory data statistical processing, it is possible to obtain a generalized mathematical model of capillary curves. The generalized model parameters $\mathbf{a}, \boldsymbol{b}$ and $\mathbf{c}$ are the characteristics of a given productive formation, and every productive formation is uniquely characterized by its own parameters. These parameters are unchanged for a specific productive formation, but different for the layers of different oil fields. The parameter a determines the wettability degree of the surface of the pore channel, that is, the waterretaining capacity of the productive formation. The parameter $\boldsymbol{b}$ characterizes the micro-heterogeneity of the channels in their size, as well as the share of the channels in the liquid filtration process. In addition, the relative phase permeabilities for the wetting (water) and nonwetting (oil, gas) phases for different water saturations are determined as well by the $\boldsymbol{b}$ parameter. The parameter $\boldsymbol{c}$ characterizes the capillary pressure curve behavior in the area of minimum values of the pore channel sizes. Thus, the generalized model of capillary pressure unequivocally characterizes the given operational facility, and the parameters of the model can be used to recognize an analogous object under development. Obviously, the experience of an analogous object developing can be confidently used in developing considered field.
\end{abstract}

\section{Key words:}

Capillary pressure curve, generalized model, grouping of facilities, capillarimetric studies, filtration-capacity properties.

\section{REFERENCES}

1. Sergeev V.L., Nguyen Ph.T.H. Models and algorithms for adaptive interpretation of combined well test data of intelligent wells. Bulletin of the Tomsk Polytechnic University. Geo Assets Engineering, 2018, vol. 329, no. 10, pp. 67-75. In Rus.

2. Mukhametshin V.V. Oil production facilities management improving using the analogy method. SOCAR Proceedings, 2020, no. 4, pp. 42-50. DOI: 10.5510/OGP20200400464. In Rus.

3. Feng Zhang, Daoyong Yang. Determination of minimum permeability plateau and characteristic length in porous media with non-Darcy flow behavior. Journal of Petroleum Science and Engineering, 2014, vol. 119, pp. 8-16. DOI: 10.1016/j.petrol.2014.04.018.

4. Jing Cheng, Dong Xiaowei, Cuid Wenhao, Dong Zhenzhen, Rena Long, Gua Xiaoyu, He Yanlong, Pu Chunsheng, Liu Xiaoyu. Artificial neural network-based time-domain interwell tracer testing for ultralow-permeability fractured reservoirs. Journal of Petroleum Science and Engineering, 2020, vol. 195, 107558, pp. 1-9. DOI: 10.1016/j.petrol.2020.107558.

5. Khormali A., Sharifov A.R., Torba D.I. Investigation of barium sulfate precipitation and prevention using different scale inhibitors under reservoir conditions. International Journal of Engineering, Transactions A: Basics, 2018, vol. 31, Iss. 10, pp. 1796-1802. DOI: 10.5829/ije.2018.31.10a.24

6. Rogachev M.K., Mukhametshin V.V., Kuleshova L.S. Improving the efficiency of using resource base of liquid hydrocarbons in Jurassic deposits of Western Siberia. Journal of Mining Institute, 2019, vol. 240, pp. 711-715. In Rus. DOI: 10.31897/PMI.2019.6.711.

7. Rogachev M.K., Mukhametshin V.V. Control and regulation of the hydrochloric acid treatment of the bottomhole zone based on field-geological data. Journal of Mining Institute, 2018, vol. 231, pp. 275-280. In Rus. DOI: 10.25515/PMI.2018.3.275. 
8. Imqam A., Bai B., Wei M., Elue H., Muhammed F.A. Use of hydrochloric acid to remove filter-cake damage from preformed particle gel during conformance-control treatments. $S P E$ Production \& Operations, 2016, vol. 31, no. 3, 11 p. DOI: 10.2118/172352-PA.

9. Galkin V.I., Ponomareva I.N., Cherepanov S.S., Filippov E.V., Martyushev D.A. New approach to the study of the results of hydraulic fracturing (on the example of bobrikovsky deposits of the Shershnevsky field). Bulletin of the Tomsk Polytechnic University. Geo Assets Engineering, 2020, vol. 331, no. 4, pp. 107-114. In Rus. DOI: 10.18799/24131830/2020/4/2598.

10. Jiang Tongwen, Yao Wei, Sun Xiongwei, Qi Chunyan, Li Xing, Xia Kaiwen, Zhang Jie, Nasseri M.H.B. Evolution of anisotropic permeability of fractured sandstones subjected to true-triaxial stresses during reservoir depletion. Journal of Petroleum Science and Engineering, 2020, 108251, pp. 1-17. DOI: 10.1016/j.petrol.2020.108251.

11. Kozyrev N.D., Vishnyakov A.Yu., Putilov I.S. Assessment of the Uncertainty Parameters Influence on the Development Indicators Forecasting. Perm Journal of Petroleum and Mining Engineering, 2020, vol. 20, no. 4, pp. 356-368. In Rus. DOI: 10.15593/2712 $8008 / 2020.4 .5$

12. Mukhametshin V.V., Kuleshova L.S. On uncertainty level reduction in managing waterflooding of the deposits with hard to extract reserves. Bulletin of the Tomsk Polytechnic University. Geo Assets Engineering, 2020, vol. 331, no. 5, pp. 140-146. In Rus. DOI $10.18799 / 24131830 / 2020 / 5 / 2644$.

13. Xinlei Sh.I., Yuniiang C.U.I., Wankun X.U., Jiansheng Zhang, Yeqin Guan. Formation permeability evaluation and productivity prediction based on mobility from pressure measurement while drilling. Petroleum Exploration and Development, 2020, vol. 47, Iss. 1, pp. 146-153. DOI: 10.1016/S1876-3804(20)60013-1.

14. Nasr-El-Din H.A., Van Domelen M.S., Sierra L., Welton Th.D. Optimization of Surfactant-based Fluids for Acid Diversion. European Formation Damage Conference. Scheveningen, The Netherlands, 30 May - 1 June 2007. 11 p. DOI: 10.2118/107687MS.

15. Yakupov R.F., Mukhametshin V.Sh., Khakimzyanov I.N., Trofimov V.E. Optimization of reserve production from water oil zones of D3ps horizon of Shkapovsky oil field by means of horizontal wells. Georesursy, 2019, vol. 21, no. 3, pp. 55-61. In Rus. DOI: 10.18599/grs.2019.3.55-61.

16. Ghareb Hamada, Veronique Joseph. Developed correlations between sound wave velocity and porosity, permeability and mechanical properties of sandstone core samples. Petroleum Research, 2020, vol. 5, Iss. 4, pp. 326-338. DOI: 10.1016/j.ptlrs.2020.07.001.

17. Akhmetov R.T., Kuleshova L.S., Mukhametshin V.V. Application of the Brooks-Corey model in the conditions of lower cretaceous deposits in terrigenous reservoirs of Western Siberia. IOP Conference Series: Materials Science and Engineering (MEACS 2018 - International Conference on Mechanical Engineering, Automation and Control Systems), 2019, vol. 560, 012004, pp. 1-4. DOI: $10.1088 / 1757-899$ X/560/1/012004.

18. Ali M.A., Umer R., Khan K.A., Cantwell W.J. Application of X-ray computed tomography for the virtual permeability prediction of fiber reinforcements for liquid composite molding processes: A review. Composites Science and Technology, 2019, vol. 184, 107828, pp. $1-20 . \quad$ DOI: 10.1016/j.compscitech.2019.107828.

19. Jun He, Kegang Ling. Measuring permeabilities of Middle-Bakken samples using three different methods. Journal of Natural Gas Science and Engineering, 2016, vol. 31, pp. 28-38. DOI: 10.1016/j.jngse.2016.03.007.

20. Akhmetov R.T., Mukhametshin V.V., Kuleshova L.S., Grezina O.A., Malyshev P.M. The generalized correlating function of capillary curves and the relationship of the filtration-capacitive parameters of reservoirs in Western Siberia with the size distribution of pore channels. Journal of Physics: Conference Series (International Conference on Information Technology in Business and Industry (ITBI 2020)), 2020, vol. 1661, 012016, pp. 1-7. DOI: 10.1088/1742-6596/1661/1/012016.

21. Mikhailov N.N., Sechina L.S., Motorova K.A. Role of clay minerals in formation of the adsorption-connected oil in rockcollectors of hydrocarbonic raw materials. Georesources, geoenergetics, geopolitics, 2012, no. 1 (5), pp. 51-62. In Rus.

22. Shelepov V.V., Tyukavkina O.V. Geophysical methods for determining the reservoir properties of complexly constructed lower and middle Jurassic deposits within the oil-gas fields of the $\mathrm{Ob}$ river region. Vestnik Moskovskogo Universiteta, Seriya 4: Geologiya, 2020, no. 1, pp. 37-41. In Rus. DOI: 10.3103/S0145875220020088.

23. Sergeev V.L., Hoang D.V., Khagay D.E., Ignatenko A.V. Diagnostic criteria for identification of filtration flows during horizontal well tests. Bulletin of the Tomsk Polytechnic University. Geo Assets Engineering, 2020, vol. 331, no. 2, pp. 181-187. In Rus. DOI: $10.18799 / 24131830 / 2020 / 2 / 2504$

24. Alimkhanov R., Samoylova I. Application of data mining tools for analysis and prediction of hydraulic fracturing efficiency for the $\mathrm{BV}_{8}$ reservoir of the Povkh oil field. SPE Russian oil and gas exploration \& Production technical conference and exhibition. Moscow, Russia, October 14-16 2014. 20 p. DOI: 10.2118/171332-RU.

25. Soloviev N.N., Mukhametshin V.Sh., Safiullina A.R. Developing the efficiency of low-productivity oil deposits via internal flooding. IOP Conference Series: Materials Science and Engineering (International Conference on Extraction, Transport, Storage and Processing of Hydrocarbons \& Materials (ETSaP)), 2020, vol. 952, 012064, pp. 1-5. DOI: 10.1088/1757899X/952/1/012064.

26. Jiazheng Qin, Shiqing Cheng, Peng Li, Youwei He, Xin Lu, Haiyang Yu. Interference well-test model for vertical well with double-segment fracture in a multi-well system. Journal of Petroleum Science and Engineering, 2019, vol. 183, 106412, pp. 1-14. DOI: 10.1016/j.petrol.2019.106412.

27. Smetkina M.A., Melkishev O.A., Prisyazhnyuk M.A. Refining the values of permeability when adapting the hydrodynamic model. Perm Journal of Petroleum and Mining Engineering, 2020, vol. 20, no. 3, pp. 223-230. In Rus. DOI: 10.15593/2712-8008/2020.3.3.

28. McLachlan G.J. Discriminant analysis and statistical pattern recognition. Hoboken, New Jersey, John Wiley \& Sons, 2004. $545 \mathrm{p}$.

Received: 21 October 2021.

\section{Information about the authors}

Rasul T. Akhmetov, Cand. Sc., professor, Ufa State Petroleum Technological University, Branch of the University in the City of Oktyabrsky.

Vyacheslav V. Mukhametshin, Dr Sc., professor, Ufa State Petroleum Technological University.

Lyubov S. Kuleshova, Cand. Sc., senior lecturer, deputy director for Academic Affairs, Ufa State Petroleum Technological University, Branch of the University in the City of Oktyabrsky.

Olga A. Grezina, associate professor, senior lecturer, deputy head of the Department of Oil and Gas Field Exploration and Development, Ufa State Petroleum Technological University, Branch of the University in the City of Oktyabrsky. 